\title{
Pulse wave velocity and cardiac autonomic function in type 2 diabetes mellitus
}

\author{
Stamatina Chorepsima', Ioanna Eleftheriadou', Anastasios Tentolouris' ${ }^{1}$, loannis Moyssakis², \\ Athanasios Protogerou', Alexandros Kokkinos ${ }^{1}$, Petros P. Sfikakis ${ }^{1}$ and Nikolaos Tentolouris ${ }^{1 *}$
}

\begin{abstract}
Background: Increased carotid-femoral pulse wave velocity (PWV) has been associated with incident cardiovascular disease, independently of traditional risk factors. Cardiac autonomic dysfunction is a common complication of diabetes and has been associated with reduced aortic distensibility. However, the association of cardiac autonomic dysfunction with PWV is not known. In this study we examined the association between cardiac autonomic function and PWV in subjects with type 2 diabetes mellitus.

Methods: A total of 290 patients with type 2 diabetes were examined. PWV was measured th the carotid-femoral segment with applanation tonometry. Central mean arterial blood pressure (MBP) was determined by the same apparatus. Participants were classified as having normal $(n=193)$ or abnormal $(n=97)$ PWV values using agecorrected values. Cardiac autonomic nervous system activity was determined by measurement of parameters of heart rate variability (HRV).

Results: Subjects with abnormal PWV were older, had higher arterial blood pressure and higher heart rate than those with normal PWV. Most of the values of HRV were significantly lower in subjects with abnormal than in those with normal PWV. Multivariate analysis, after controlling for various confounding factors, demonstrated that abnormal PWW was associated independently only with peripheral MBP [odds ratio (OR) 1.049, 95\% confidence intervals $(C l) 1.015-1.085, P=0.005$ ], central MBP (OR 1.052, 95\% Cl 1.016-1.088, $P=0.004$ ), log total power (OR 0. $490,95 \% \mathrm{Cl} 0.258-0.932, P=0.030$ ) and log high frequency power (OR 0.546, 95\% Cl 0.301-0.991, $P=0.047$ ).
\end{abstract}

Conclusions: In subjects with type 2 diabetes, arterial blood pressure and impaired cardiac autonomic function is associated independently with abnormal PWV.

Keywords: Cardiac autonomic dysfunction, Pulse wave velocity, Type 2 diabetes mellitus

\section{Background}

Pulse wave velocity (PWV) is the gold-standard method for the assessment of arterial stiffness and is an independent predictor of cardiovascular morbidity and mortality [1]. Previous studies have shown that arterial stiffness is increased in people with diabetes $[2,3]$ and that PWV independently predicts mortality in this group of patients [3].

Cardiac autonomic dysfunction is a common although underestimated chronic microvascular complication of diabetes [4]. Cardiac autonomic dysfunction is a well-

\footnotetext{
* Correspondence: ntentol@med.uoa.gr

${ }^{1}$ First Department of Propaedeutic and Internal Medicine, Medical School, National and Kapodistrian University of Athens, Laiko General Hospital, 17 Agiou Thoma St, 11527 Athens, Greece

Full list of author information is available at the end of the article
}

established risk factor for cardiovascular morbidity and all-cause and cardiovascular mortality [5]. Decreased heart rate variability (HRV) and low baroreflex sensitivity (BRS) are considered to be early markers of cardiac autonomic dysfunction [6, 7].

Several studies have reported that early in the course of type 1 diabetes impaired cardiac autonomic function and arterial stiffness are strongly associated [8, 9]. Moreover, patients without diabetes but with primary autonomic failure have stiffer aortas when compared with healthy age- and sex-matched control individuals [10]. These findings imply that there is a pathophysiological link between cardiac autonomic dysfunction and arterial stiffness and that the preservation of the elastic 
properties of the arteries strongly depends on the integrity of the autonomic nervous system.

Recent guidelines emphasize that use of normal and abnormal PWV values according to age represent a critical step in the implementation of PWV as a clinical tool for identification of people at higher cardiovascular risk [11]. It is known for several decades that people with type 2 diabetes (T2DM) have a 2-4 higher relative risk for cardiovascular disease [12]. Previous studies in patients with T2DM have shown that arterial stiffness is associated with age, blood pressure, duration of diabetes and cardiac autonomic dysfunction [2, 13, 14]. However, no data exist on the potential association between abnormal PWV, defined according to recent guidelines, impaired cardiac autonomic function and classical risk factors for atherosclerosis in people with T2DM.

Based on the above literature data, the research hypothesis we examined in this study is that impaired cardiac autonomic function is associated with abnormal PWV in people with T2DM, when diabetes-related and classical risk factors for atherosclerosis are taken into consideration.

\section{Methods}

\section{Participants}

A total of 290 patients with T2DM were recruited consecutively from the diabetes center of our hospital. Individuals were recruited if they were adults of 18-75 years of age and had been diagnosed with T2DM according to the American Diabetes Association criteria [15]. Exclusion criteria were atrial flutter or fibrillation, pacemaker, history of severe liver or kidney disease [estimated glomerular filtration rate (eGFR) $<30 \mathrm{ml} / \mathrm{min} /$ $1.73 \mathrm{~m}^{2}$ ], heart failure of stage III and IV and acute illness or hypoglycemia in the previous $24 \mathrm{~h}$. In addition, in order to minimize the confounding effect of medications on parameters of HRV, we excluded patients on antiarrhythmic drugs other than b-blockers or drugs with an effect on cardiac autonomic nervous system activity like antidepressants and antihistamines. The study was approved by the ethics committee of our hospital and was conducted according to the principles of the Declaration of Helsinki [16]. The aim of the study was clearly explained to all individuals and written informed consent was obtained before participation in the study.

\section{Procedures}

This is a cross-sectional study and the examination was carried out between 07.30 and $09.30 \mathrm{~h}$ in the morning in a room of stable temperature $\left(22-24{ }^{\circ} \mathrm{C}\right)$. All participants abstained from any food or drink except for water for $12 \mathrm{~h}$ before the study and they received their medications after the end of the study. A complete physical examination was performed and established questionnaires were used to evaluate history of previous disease, current disease and use of medications. The participants were also questioned about their smoking habits and were characterized as exsmokers if they have been given up smoking for more than 2 years, non-smokers or current smokers. Height, weight as well as waist circumference were measured in light clothing and body mass index (BMI, in $\mathrm{kg} / \mathrm{m}^{2}$ ) was calculated. Blood pressure was measured at the brachial artery using an appropriate cuff size three times at 5-min intervals with the participant in the sitting position. The mean value of the last two measurements was used in the analysis. Arterial hypertension was defined according to current guidelines [17] if systolic blood pressure (SBP) was $\geq 140 \mathrm{mmHg}$ and/or diastolic blood pressure (DBP) $\geq 90 \mathrm{mmHg}$, or if patients were on antihypertensive treatment. Peripheral mean blood pressure (pMBP) was calculated from SBP and $\mathrm{DBP}$ as $\mathrm{MBP}=\mathrm{DBP}+0.4$ (SBP-DBP) [18].

Patients on treatment with lipid lowering agents and those having total cholesterol $>200 \mathrm{mg} / \mathrm{dl}$ and/or low-density lipoprotein (LDL) cholesterol $>70 \mathrm{mg} / \mathrm{dl}$ for patients with a history of cardiovascular disease or $>100 \mathrm{mg} / \mathrm{dl}$ for participants without history of cardiovascular disease and/or high density lipoprotein (HDL) cholesterol $<40 \mathrm{mg} / \mathrm{dl}$ for men or $<50 \mathrm{mg} / \mathrm{dl}$ women and/or fasting triglyceride levels $>150 \mathrm{mg} / \mathrm{dl}$ were considered as having dyslipidemia. Coronary artery disease was defined as a history of angina, myocardial infarction, percutaneous transluminal coronary angioplasty or coronary artery bypass grafting. Peripheral arterial disease was defined as a history of intermittent claudication, revascularization procedures at the aorta or the lower limbs or as an ankle brachial pressure index $<0.90$ [19]. Cerebrovascular disease was defined as a history of stroke or revascularization at the carotid arteries.

Diagnosis of diabetic peripheral neuropathy was based on neuropathy symptom score (NSS) and neuropathy disability score (NDS). The criteria for the diagnosis of diabetic peripheral neuropathy were NDS $\geq 6$ irrespective of the NSS values, or NDS $=3-5$ with NSS $\geq 5$ [20].

Blood was drawn early in the morning after $12 \mathrm{~h}$ of fasting. Serum lipids (total cholesterol, HDL, triglycerides) and creatinine were measured enzymatically on an automated analyzer. LDL levels were calculated using the Friedewald's formula [21]. eGFR was calculated according to Modification of Diet in Renal Disease (MDRD) formula [22]. Nephropathy was defined as an eGFR $<60 \mathrm{ml} / \mathrm{min} / 1.73 \mathrm{~m}^{2}$ and/or as the presence of microalbuminuria or proteinuria.

\section{Assessment of pulse wave velocity (PWV)}

PWV was measured by applanation tonometry with a validated noninvasive device (SphygmoCor, AtCor Medical, Sydney, Australia). PWV was calculated from measurements 
of pulse transit time and the distance traveled between the common carotid artery and the common femoral artery. The distance measurements were taken with a measuring tape by substracting the distance from the suprasternal notch to the carotid from the suprasternal notch to the femoral artery at the sensor location. Patients were classified as having normal or abnormal PWV values according to their age; values of PWV above the $90^{\text {th }}$ percentile were considered abnormal [11]. In addition, the SphygmoCor device, that uses radial tonometry via a high-fidelity probe to derive aortic blood pressure from a validated transfer function after calibration, was used for determination of central blood pressures [23].

\section{Assessment of heart rate variability (HRV)}

Short-term analysis of the HRV was performed in all participants using the computer-aided examination and evaluation system VariaCardio TF5 (Medical Research Limited, Leeds, UK) [4, 24]. Frequency domain parameters of HRV were obtained after a 5-min recording at each interval on a 256 beat-window basis. Data were analyzed by Fast Fourier Transform modified by the coarse-graining algorithm. Each dataset was filtered automatically by excluding recorded artifacts using a recognition algorithm. Parameters of the frequencydomain were observed within the high frequency (HF) band $(0.15$ to $0.50 \mathrm{~Hz})$ and within the low frequency (LF) band (0.05 to $0.15 \mathrm{~Hz}$ ). Power in LF range (0.04$0.15 \mathrm{~Hz})$ and power in $\mathrm{HF}$ range $(0.15-0.40 \mathrm{~Hz})$ were recorded. Total power (TP) (frequency range: $\leq 0.40 \mathrm{~Hz}$ ), the sum of all the components, was also obtained. Subsequently, the ratio LF/HF was calculated. In addition, the following time-domain parameters of HRV were determined: normal-to-normal RR interval (NN), standard deviation of all normal-to-normal RR intervals (SDNN) and square root of the mean of the squares of successive differences between adjacent $\mathrm{NN}$ intervals ( $\mathrm{r}$-MSDD). Vagal activity is the major contributor to the HF component and to the time-domain parameters, while LF reflects both sympathetic and vagal activity. TP represents the sum of all the frequency components, whereas the ratio LF/HF is considered to mirror sympathovagal balance [7].

\section{Assessment of baroreflex sensitivity (BRS)}

BRS estimation was performed in all participants by the sequence method using the Barocor System (AtCor Medical, Sydney, Australia), as previously described [24]. In summary, electrocardiographic signal with a three lead electrocardiogram and beat-to-beat blood pressure were continuously and simultaneously recorded for 20 min. Time series of inter-beat (RR) intervals and systolic blood pressure were analyzed by the BaroCor System Software to identify sequences in which systolic blood pressure and $\mathrm{RR}$ interval increased or decreased concurrently over at least three cardiac cycles. Lag 0 value of central BRS was selected for each participant measurement.

\section{Statistical methods}

Statistical analysis was performed using the SPSS 22.0 statistical package (IBM SPSS software version 22.0 for Windows, Armonk, NY, USA). All data were assessed for normal distribution of their values using the Kolmogorov-Smirnov test. As BRS and parameters of HRV were skewed, their values were log-transformed to improve normality for statistical testing. Student's $t$-test and the Mann-Whitney test were used to assess differences in normally and non-normally distributed continuous variables between the studied groups, while the Chi-square test was used for categorical variables. Univariate and multivariate logistic regression analyses were performed to examine for associations between PWV stratified as normal or abnormal using age- and method-corrected reference values [11] and the studied parameters. Variables that were found to differ significantly between participants with normal or abnormal PWV in the univariate analysis were entered in the models of multivariate logistic regression analysis. Because HRV modalities were highly correlated, several models of multivariate analysis were created for each one of the HRV parameters to avoid multi-collinearity. Moreover, to avoid multi-collinearity, $\mathrm{pMPB}$ and central MBP (cMBP) were entered in the models of multivariate logistic regression analyses consecutively. $P$ values $<0.05$ (two-tailed) were considered statistically significant.

\section{Results}

The demographic and clinical characteristics of the study participants classified according to their PWV status are depicted in Table 1. Patients with abnormal PWV were older, had higher arterial blood pressure $(P<0.001)$ and higher heart rate $(P<0.001)$ than those with normal PWV; additionally, they were treated more often with insulin $(P=0.033)$ and less often with diuretics. Participants with normal and abnormal PWV did not differ in terms of gender, BMI, duration of diabetes, waist circumference, $\mathrm{HbA}_{1 \mathrm{c}}$, smoking status, lipid profile, treatment for dyslipidemia or hypertension, use of antiplatelets, prevalence of macrovascular complications and prevalence of retinopathy or nephropathy, except for peripheral neuropathy which was more common in participants with abnormal $\operatorname{PWV}(P=0.030)$.

The values of log TP, log power HF, log r-MSDD and the log NN mean of the HRV were lower in participants with abnormal PWV than in those with normal PWV $(P=0.010, P=0.014, P=0.034$ and $P=0.042$, respectively). The values of the $\log$ power LF, the ratio 
Table 1 Demographic, clinical characteristics and laboratory parameters of the study participants

\begin{tabular}{|c|c|c|c|}
\hline & \multicolumn{2}{|l|}{$n=290$} & \multirow[b]{2}{*}{$P$ value } \\
\hline & $\begin{array}{l}\text { normal PWV } \\
(n=193)\end{array}$ & $\begin{array}{l}\text { abnormal PWV } \\
(n=97)\end{array}$ & \\
\hline PWV (m/s) & $9.1 \pm 1.7$ & $12.6 \pm 2.5$ & $<0.001^{*}$ \\
\hline PWV (m/s) & $9.0[8.0,10.4]$ & $12.8[10.4,14.1]$ & $<0.001^{* *}$ \\
\hline Age (years) & $60.5 \pm 9.5$ & $63.8 \pm 7.8$ & $0.004^{*}$ \\
\hline Duration of diabetes (years) & $10.0[5.0,18.0]$ & $9.0[3.0,16.0]$ & $0.130^{* *}$ \\
\hline Male gender n (\%) & $114(59.1)$ & $49(50.5)$ & $0.166^{* * *}$ \\
\hline Height (m) & $1.65 \pm 0.10$ & $1.66 \pm 0.10$ & $0.223^{*}$ \\
\hline Weight (kg) & $84.5 \pm 16.3$ & $85.6 \pm 16.3$ & $0.577^{*}$ \\
\hline Body mass index $\left(\mathrm{kg} / \mathrm{m}^{2}\right)$ & $31.1 \pm 4.8$ & $30.9 \pm 4.8$ & $0.755^{*}$ \\
\hline Waist circumference $(\mathrm{cm})$ & $104.3 \pm 11.6$ & $105.6 \pm 12.1$ & $0.395^{*}$ \\
\hline Current smoking n (\%) & $40(20.7)$ & $20(20.6)$ & $0.983^{* * *}$ \\
\hline Pack-years & $37.5[21.3,65.3]$ & $40[32.0,50.0]$ & $0.070^{* *}$ \\
\hline SBP $(\mathrm{mmHg})$ & $139.5 \pm 18.4$ & $148.9 \pm 19.8$ & $<0.001^{*}$ \\
\hline $\mathrm{DBP}(\mathrm{mmHg})$ & $75.7 \pm 9.4$ & $81.4 \pm 10.2$ & $<0.001^{*}$ \\
\hline Peripheral MBP (mmHg) & $97.5 \pm 10.6$ & $104.8 \pm 11.9$ & $<0.001^{*}$ \\
\hline Central MBP (mmHg) & $94.0 \pm 10.6$ & $100.9 \pm 11.7$ & $<0.001^{*}$ \\
\hline $\mathrm{HR}(\mathrm{bpm})$ & $66.4 \pm 9.3$ & $70.6 \pm 9.6$ & $<0.001^{*}$ \\
\hline Hypertension n (\%) & $145(75.1)$ & $76(78.4)$ & $0.543^{* * *}$ \\
\hline \multicolumn{4}{|l|}{ Antihypertensive drugs n (\%) } \\
\hline Use of ACEi or ARBs n (\%) & $121(62.7)$ & $62(63.9)$ & $0.839^{* * *}$ \\
\hline Use of CCBs n (\%) & $58(30.1)$ & $21(21.6)$ & $0.129^{* * *}$ \\
\hline Use of $\beta$-blockers n (\%) & $59(30.6)$ & $30(30.9)$ & $0.950^{* * *}$ \\
\hline Use of diuretics n (\%) & $70(36.3)$ & $24(24.7)$ & $0.048^{* * *}$ \\
\hline Total cholesterol (mmol/L) & $4.40 \pm 0.84$ & $4.37 \pm 1.18$ & $0.491^{*}$ \\
\hline HDL-cholesterol (mmol/L) & $1.19 \pm 0.29$ & $1.21 \pm 0.32$ & $0.571^{*}$ \\
\hline LDL-cholesterol (mmol/L) & $2.55 \pm 0.91$ & $2.53 \pm 0.85$ & $0.571^{*}$ \\
\hline Triglycerides (mmol/L) & $1.33[1.02,1.78]$ & $1.42[1.12,2.11]$ & $0.069^{* *}$ \\
\hline Dyslipidemia n (\%) & $155(80.3)$ & $84(86.6)$ & $0.185^{* * *}$ \\
\hline Treatment with statins n (\%) & $154(79.8)$ & $80(82.5)$ & $0.585^{* * *}$ \\
\hline Cardiovascular disease n (\%) & $45(23.3)$ & $24(24.7)$ & $0.788^{* * *}$ \\
\hline Coronary heart disease & $45(23.3)$ & $15(15.5)$ & $0.119^{* * *}$ \\
\hline Peripheral arterial disease & $22(11.4)$ & $11(11.3)$ & $0.988^{* * *}$ \\
\hline Stroke & $11(5.7)$ & $3(3.1)$ & $0.329^{* * *}$ \\
\hline Treatment with antiplatelets n (\%) & $100(51.8)$ & $56(60.2)$ & $0.340^{* * *}$ \\
\hline Glucose (mmol/L) & $8.1 \pm 2.6$ & $8.4 \pm 2.9$ & $0.335^{*}$ \\
\hline $\mathrm{HbA}_{1 c}(\%)$ & $7.1[6.5,7.9]$ & $7.1[6.5,8.0]$ & $0.725^{* *}$ \\
\hline \multicolumn{4}{|l|}{ Antidiabetic treatment n (\%) } \\
\hline Oral medications & $122(63.2)$ & $56(57.7)$ & $0.366^{* * *}$ \\
\hline Insulin & $13(6.7)$ & $14(14.4)$ & $0.033^{* * *}$ \\
\hline Both & $58(30.1)$ & $27(27.8)$ & $0.696^{* * *}$ \\
\hline Nephropathy n (\%) & $66(34.2)$ & $40(41.2)$ & $0.240^{* * *}$ \\
\hline eGFR $\left(\mathrm{ml} / \mathrm{min} / 1.73 \mathrm{~m}^{2}\right)$ & $75.3 \pm 21.2$ & $70.9 \pm 27.6$ & $0.308^{*}$ \\
\hline
\end{tabular}


Table 1 Demographic, clinical characteristics and laboratory parameters of the study participants (Continued)

\begin{tabular}{llll}
\hline \multicolumn{1}{c}{ Microalbuminuria $\mathrm{n}(\%)$} & $38(19.7)$ & $19(19.6)$ & $0.984^{* * *}$ \\
Peripheral neuropathy $\mathrm{n}(\%)$ & $31(16.1)$ & $26(26.8)$ & $0.030^{* * *}$ \\
Retinopathy $\mathrm{n}(\%)$ & $29(15.0)$ & $22(22.6)$ & $0.106^{* * *}$ \\
\hline
\end{tabular}

Data are $\mathrm{n}(\%)$, means \pm SD (standard deviation), median value $(25,75$ percentile)

$P W V$ pulse wave velocity, SBP systolic blood pressure, DBP diastolic blood pressure, $M B P$ mean blood pressure, $H R$ heart rate, $A C E i$ angiotensin converting enzyme inhibitors, $A R B s$ angiotensin II receptor antagonists, $C C B s$ calcium channel blockers, $H D L$ high density lipoprotein, $L D L$ low density lipoprotein, $H b A_{1 c}$ glycated hemoglobin, eGFR estimated glomerular filtration rate

${ }^{*} p$ values for comparisons between groups by Independent samples t-test

${ }^{* *} p$ values for comparisons between groups by Mann-Whitney $\mathrm{U}$ test

*** $p$ values for comparisons between groups by Chi-squared test

LF/HF, the $\log$ SDNN and the log BRS did not differ between the two groups (Table 2).

Univariate logistic regression analysis demonstrated that there were significant associations between abnormal PWV, SBP, DBP, pMBP and cMBP, heart rate, triglycerides, peripheral neuropathy, and most of the parameters of HRV; no significant association was found between log BRS and PWV (Table 3). Multivariate logistic regression analysis, after adjustment for the effect of age, gender, heart rate and triglycerides, demonstrated that the odds of abnormal PWV were associated significantly and independently only with higher pMBP, cMBP and worse cardiac autonomic nervous system function indices such as lower log TP and lower log HF, while there was a trend for association with lower log r-MSDD (Table 3).

\section{Discussion}

In the present study, we showed that beyond blood pressure, impaired cardiac autonomic function assessed by determination of HRV was a significant determinant of

Table 2 The values of parameters of heart rate variability and of baroreflex sensitivity stratified according to the pulse wave velocity status

\begin{tabular}{llll}
\hline & $\begin{array}{l}\text { normal PWV } \\
(n=193)\end{array}$ & $\begin{array}{l}\text { abnormal PW } \\
(n=97)\end{array}$ & $P$ value \\
\hline Log total Power $\left(\mathrm{msec}^{2}\right)$ & $2.44 \pm 0.58$ & $2.17 \pm 0.62$ & $0.010^{* *}$ \\
Log Power HF $\left(\mathrm{msec}^{2}\right)$ & $2.03 \pm 0.65$ & $1.75 \pm 0.61$ & $0.014^{* *}$ \\
Log Power LF $\left(\mathrm{msec}^{2}\right)$ & $2.01 \pm 0.63$ & $1.82 \pm 0.69$ & $0.087^{* *}$ \\
LF/HF & $1.1[0.5,1.9]$ & $1.1[0.6,2.5]$ & $0.399^{*}$ \\
Log NN mean (msec) & $2.96 \pm 0.06$ & $2.93 \pm 0.06$ & $0.042^{* *}$ \\
Log SDNN (msec) & $1.48 \pm 0.25$ & $1.40 \pm 0.29$ & $0.073^{* *}$ \\
Log r-MSDD (msec) & $2.65 \pm 0.65$ & $2.41 \pm 0.66$ & $0.034^{* *}$ \\
Log BRS (msec/mmHg) & $0.76 \pm 0.24$ & $0.72 \pm 027$ & $0.368^{* *}$ \\
\hline
\end{tabular}

Data are $\mathrm{n}(\%)$, means $\pm \mathrm{SD}$ (standard deviation), median value (25,

75 percentile)

$P W V$ pulse wave velocity, $L o g$ logarithmic value, $H F$ high frequency, $L F$ low frequency, $N N$ normal-to-normal RR interval, SDNN standard deviation of all normal-to-normal RR intervals, $r-M S D D$ square root of the mean of the squares of successive differences between adjacent NN intervals, BRS baroreflex sensitivity

${ }^{*} p$ values for comparisons between groups by Mann-Whitney $\mathrm{U}$ test

${ }^{* *} p$ values for comparisons between groups by Independent samples t-test abnormal PWV in people with T2DM. Furthermore, lower values of the frequency-dependent domains of the HRV were independently associated with higher odds of abnormal PWV.

The findings of our study are in accordance with those of previous published studies that investigated the association between cardiac autonomic dysfunction and aortic stiffness in patients with T2DM [13, 14]. Our group described previously that patients with T2DM and cardiac autonomic neuropathy had reduced aortic distensibility, an index of aortic stiffness, when compared with patients with T2DM without cardiac autonomic neuropathy, while duration of diabetes and presence of cardiac autonomic neuropathy were the main determinants of reduced aortic distensibility [13]. Another study also demonstrated a significant association between autonomic neuropathy, assessed using HRV, and systemic arterial compliance as well as PWV in patients with T2DM [14]. It should be taken into account that the diabetic population in these two studies was a selected group without macrovascular disease or hypertension, whereas in our study we did not exclude patients with macrovascular complications. Thus, our sample is more representative of the general diabetic population. In addition, the present study is the first to use the agecorrected reference values for PWV.

The pathophysiological link between aortic stiffness and autonomic dysfunction and whether impaired cardiac autonomic function induces arterial stiffening or whether increased arterial stiffness leads to the impairment of the autonomic function remains obscure. Both arterial stiffness and cardiac autonomic dysfunction share common pathogenetic pathways including chronic hyperglycemia and hyperinsulinemia, formation of advanced glycation end-products (AGEs) and protein kinace $\mathrm{C}$ activation, low grade inflammation and endothelial dysfunction [2]. One hypothesis is that impaired cardiac autonomic function results in increased arterial stiffness. An explanation could be that patients with cardiac autonomic neuropathy present more often with calcification of the tunica media of the arterial wall [25]. It is noteworthy that the main determinant of the extent of arterial calcification is the severity of autonomic 
Table 3 Associations between the studied parameters and abnormal pulse wave velocity in participants with type 2 diabetes

\begin{tabular}{|c|c|c|c|}
\hline & OR & $95 \% \mathrm{Cl}$ & $P$ value \\
\hline \multicolumn{4}{|l|}{ Univariate logistic regression analysis } \\
\hline Age (years) & 0.956 & $0.936-1.033$ & 0.504 \\
\hline Gender (men vs. women) & 0.707 & $0.433-1.155$ & 0.167 \\
\hline Diabetes duration (years) & 0.979 & $0.951-1.008$ & 0.150 \\
\hline Height (m) & 4.513 & $0.400-50.860$ & 0.223 \\
\hline Body mass index $\left(\mathrm{kg} / \mathrm{m}^{2}\right)$ & 0.992 & $0.942-1.044$ & 0.754 \\
\hline Waist circumference $(\mathrm{cm})$ & 1.009 & $0.988-1.031$ & 0.394 \\
\hline Current smoking n (\%) & 0.994 & $0.544-1.815$ & 0.983 \\
\hline SBP $(\mathrm{mmHg})$ & 1.026 & $1.012-1.040$ & $<0.001$ \\
\hline $\mathrm{DBP}(\mathrm{mmHg})$ & 1.065 & $1.035-1.095$ & $<0.001$ \\
\hline Peripheral MBP (mmHg) & 1.062 & $1.037-1.089$ & $<0.001$ \\
\hline Central MBP (mmHg) & 1.059 & $1.033-1.086$ & $<0.001$ \\
\hline $\mathrm{HR}(\mathrm{bpm})$ & 1.049 & $1.021-1.077$ & 0.001 \\
\hline Total cholesterol (mmol/L) & 0.785 & $0.626-1.285$ & 0.136 \\
\hline HDL-cholesterol (mmol/L) & 0.784 & $0.339-1.814$ & 0.569 \\
\hline LDL-cholesterol (mmol/L) & 0.571 & $0.791-1.138$ & 0.571 \\
\hline Triglycerides (mmol/L) & 0.674 & $0.496-0.916$ & 0.012 \\
\hline Treatment with statins (yes vs. no) & 0.538 & $0.326-1.386$ & 0.115 \\
\hline Glucose (mmol/L) & 0.956 & $0.874-1.047$ & 0.335 \\
\hline $\mathrm{HbA}_{1 \mathrm{c}}(\%)$ & 0.996 & $0.809-1.227$ & 0.971 \\
\hline Nephropathy (yes vs. no) & 1.422 & $0.854-2.367$ & 0.176 \\
\hline Peripheral neuropathy (yes vs. no) & 2.018 & $1.111-3.667$ & 0.021 \\
\hline Log Power LF $\left(\mathrm{msec}^{2}\right)$ & 0.625 & $0.364-1.074$ & 0.089 \\
\hline Log Power HF $\left(\mathrm{msec}^{2}\right)$ & 0.499 & $0.284-0.878$ & 0.016 \\
\hline Log Total Power $\left(\mathrm{msec}^{2}\right)$ & 0.452 & $0.244-0.838$ & 0.012 \\
\hline LF/HF & 1.117 & $0.901-1.386$ & 0.312 \\
\hline Log NN mean (msec) & 0.004 & $0.000-0.866$ & 0.044 \\
\hline Log SDNN (msec) & 0.295 & $0.077-1.130$ & 0.075 \\
\hline Log r-MSDD (msec) & 0.556 & $0.321-0.963$ & 0.036 \\
\hline Log BRS (msec/mmHg) & 0.529 & $0.133-2.105$ & 0.366 \\
\hline \multicolumn{4}{|l|}{ Multivariate logistic regression analyses ${ }^{\mathrm{a}}$} \\
\hline \multicolumn{4}{|l|}{ Model 1} \\
\hline Central MBP (mmHg) & 1.052 & $1.016-1.088$ & 0.004 \\
\hline Log Total Power $\left(\mathrm{msec}^{2}\right)$ & 0.490 & $0.258-0.932$ & 0.030 \\
\hline \multicolumn{4}{|l|}{ Model 2} \\
\hline Central MBP $(\mathrm{mmHg})$ & 1.050 & $1.015-1.087$ & 0.005 \\
\hline Log Power HF $\left(\mathrm{msec}^{2}\right)$ & 0.546 & $0.301-0.991$ & 0.047 \\
\hline \multicolumn{4}{|l|}{ Model 3} \\
\hline Central MBP (mmHg) & 1.053 & $1.017-1.091$ & 0.004 \\
\hline Log r-MSDD (msec) & 0.572 & $0.319-1.024$ & 0.060 \\
\hline
\end{tabular}

$O R$ odds ratio, $C l$ confidence interval, $S B P$ systolic blood pressure, $D B P$ diastolic blood pressure, $H R$ heart rate, $H D L$ high density lipoprotein, $L D L$ low density lipoprotein $H b A_{1 c}$ glycated hemoglobin, $L F$ low frequency, Log logarithmic value, $H F$ high frequency, NN normal-to-normal RR interval, SDNN standard deviation of all normalto-normal RR intervals, $r-M S D D$ square root of the mean of the squares of successive differences between adjacent NN intervals, BRS baroreflex sensitivity

${ }^{a}$ After adjustment in addition for age, gender, heart rate and triglycerides. Gender, current smoking status, treatment with statins, nephropathy and peripheral neuropathy (yes vs. no) were analyzed as categorical variables; all the other variables were analyzed as continuous variables in both univariate and multivariate analysis. When central mean (cMBP) and peripheral mean arterial blood pressure (pMBP) were used in turn in the models of multivariate logistic regression analyses, the results were not affected significantly neuropathy [25]. On the other hand, arterial calcification has been suggested as an important determinant of arterial stiffness according to findings in humans and experimental models [26]. These data reveal that calcification of the arterial wall may be an additional common pathophysiological pathway that could explain the relationship between impaired cardiac autonomic function and arterial stiffness.

Another explanation could be that cardiac autonomic dysfunction may affect the elasticity of the arterial wall by changing the smooth muscle tone of large arteries $[8,27]$. Interestingly, people without diabetes but with primary autonomic failure have been found to have stiffer aortas when compared with healthy control individuals [10]. Although this explanation is rather difficult to be proven in humans, experimental studies have shown that sympathectomized rats exhibit a significant reduction in the elastic properties of the aorta when compared with animals with intact sympathetic ganglia [28]. In humans on the other hand, high sympathetic activity has been associated with arterial stiffness in hypertensive patients with and without T2DM, as well as in healthy individuals. Increases in heart rate per se may lead to arterial stiffening independently of changes in activity of the autonomic nervous system [8]. Nevertheless, in the present study the association between autonomic dysfunction and arterial stiffness was not mediated by an increase in heart rate.

The other hypothesis is that arterial stiffness may lead to cardiac autonomic dysfunction via impairment of baroreceptor function induced by stiffening of the arterial wall [29]. To our knowledge, no literature data exists so far on the relationship between BRS and PWV in people with T2DM. Several studies have found a significant association between low BRS and increased arterial stiffness in patients with congestive heart failure [30], in older subjects [29] and in chronic hemodialysis patients [31]. However, in our study, no difference in central BRS was observed between participants with abnormal and normal PWV. This finding may imply that diabetes per se is a strong factor affecting BRS and outweighs the potential effect of other factors on BRS.

We did not find significant associations between PWV and conventional risk factors like age, smoking habits, microalbuminuria and lipid profile. Moreover, no associations between PWV and macrovascular complications, glycemic control or gender were observed. Our findings are in line with those of a systematic review reporting that, with the exception of age and hypertension, PWV was largely independent of classic risk factors for atherosclerosis, including gender, smoking and lipids [32]. It was suggested that in the early phases of atherosclerosis increased arterial stiffness is caused not by the atherosclerotic process itself and the formation of the atherosclerotic 
plaque, for which gender, smoking and lipids are powerful risk factors, but by an alternative pathophysiological mechanism, in which increased blood pressure is one of the most important factors. Although age is a strong determinant of PWV in the general population [11], we did not find any association between age and abnormal PWV. It could be hypothesized that the presence of diabetes per se has a cardinal impact on arterial stiffness, overcoming the potential effect of other factors [11]. However, it should be noted that almost $80 \%$ of the participants in our study were on statin treatment, while more than $60 \%$ received antihypertensive medications and these factors may have influenced our results.

Increasing evidence suggests that central blood pressure may be a more accurate indicator of end organ damage and cardiovascular risk than brachial blood pressure in specific groups of patients, including individuals with T2DM [33]. A recent meta-analysis reported that central compared with brachial SBP was more closely associated with PWV [33]. We also found that although both pMBP and cMBP were independently associated with abnormal PWV, the odds for abnormal PWV were slightly higher for cMBP in comparison with pMBP [odds ratio (OR) 1.052, 95\% confidence interval (CI) $1.016-1.088, P=0.004$ vs. OR $1.049,95 \%$ CI $1.015-$ 1.085, $P=0.005]$.

The strength of our study is that it is the first to investigate the association between impaired cardiac autonomic function and abnormal PWV using age-corrected values. A limitation is, however, the cross-sectional design that does not allow determination of a causal relationship between PWV and cardiac autonomic function. Although a non-causal association cannot be ruled out, causality could only be determined if the question of which of the two events (impaired cardiac autonomic function or arterial stiffening) appears first could be answered [27]. Another limitation is that we did not recruit participants without diabetes as a control group to investigate potential differences in the associations of PVW with cardiac autonomic dysfunction between persons with and without T2DM. However, cardiac autonomic dysfunction is not common in persons without diabetes.

\section{Conclusions}

The present study has demonstrated that blood pressure and impaired cardiac autonomic function are the main determinants of abnormal PWV in people with T2DM, while the association between impaired cardiac autonomic function and arterial stiffness is not mediated by low BRS or increased heart rate. Our findings suggest that cardiac autonomic nervous system activity influences arterial stiffness and it should be monitored and reported in studies examining factors affecting PWV.

\section{Abbreviations}

AGEs: Advanced glycation end-products; BMI: Body mass index; BRS: Baroreflex sensitivity; CAD: Cardiac autonomic dysfunction; Cl: Confidence interval; CMBP: Central mean blood pressure; DBP: Diastolic blood pressure; eGFR: Estimated glomerular filtration rate; HDL: High density lipoprotein; HF: High frequency; HRV: Heart rate variability; LDL: Low-density lipoprotein; LF: Low frequency; MDRD: Modification of Diet in Renal Disease; NDS: Neuropathy disability score; NN: Normal-to-normal RR interval; NSS: Neuropathy symptom score; OR: Odds ratio; pMBP: Peripheral mean blood pressure; PWV: Pulse wave velocity; r-MSDD: Square root of the mean of the squares of successive differences between adjacent NN intervals; SBP: Systolic blood pressure; SDNN: Standard deviation of all normal-tonormal RR intervals; T2DM: Type 2 diabetes; TP: Total power

\section{Acknowledgement}

Not applicable.

Funding

No funding received.

\section{Availability of data and materials}

The datasets used and/or analyzed during the current study are available from the corresponding author on reasonable request.

\section{Authors' contributions}

SC, IE, AT, IM, AP, AK, PS and NT all met the ICMJE criteria for authorship. SC, $I E, A T, I M, A P, A K, P S$ and NT were involved in the design of the study, interpretation of results and writing of the manuscript. SC, IE, AT and IM participated in data acquisition and AT, IE and NT conducted the statistical analysis. All authors read and approved the final manuscript.

\section{Competing interests}

The authors declare that they have no competing interests.

Consent for publication

Not applicable.

\section{Ethics approval and consent to participate}

The study was approved by the ethics committee of Laiko General Hospital and written informed consent was obtained from all individuals before participation in the study.

\section{Publisher's Note}

Springer Nature remains neutral with regard to jurisdictional claims in published maps and institutional affiliations.

\section{Author details}

${ }^{1}$ First Department of Propaedeutic and Internal Medicine, Medical School, National and Kapodistrian University of Athens, Laiko General Hospital, 17 Agiou Thoma St, 11527 Athens, Greece. ²Department of Cardiology, Laiko General Hospital, Athens, Greece.

Received: 11 March 2017 Accepted: 15 May 2017

Published online: 19 May 2017

\section{References}

1. Vlachopoulos C, Aznaouridis K, Stefanadis C. Prediction of cardiovascular events and all-cause mortality with arterial stiffness: a systematic review and meta-analysis. J Am Coll Cardiol. 2010;55:1318-27.

2. Stehouwer $C D$, Henry RM, Ferreira I. Arterial stiffness in diabetes and the metabolic syndrome: a pathway to cardiovascular disease. Diabetologia. 2008:51:527-39.

3. Cruickshank K, Riste L, Anderson SG, et al. Aortic pulse-wave velocity and its relationship to mortality in diabetes and glucose intolerance: an integrated index of vascular function? Circulation. 2002;106:2085-90.

4. Vinik Al, Ziegler D. Diabetic cardiovascular autonomic neuropathy. Circulation. 2007:115:387-97.

5. Spallone V, Ziegler D, Freeman R, et al. Cardiovascular autonomic neuropathy in diabetes: clinical impact, assessment, diagnosis, and management. Diabetes Metab Res Rev. 2011;27:639-53. 
6. Rosengard-Barlund M, Bernardi L, Sandelin A, et al. Baroreflex sensitivity and its response to deep breathing predict increase in blood pressure in type 1 diabetes in a 5-year follow-up. Diabetes Care. 2011;34:2424-30.

7. Task Force of the European Society of Cardiology and the North American Society of Pacing and Electrophysiology. Heart rate variability: standards of measurement, physiological interpretation and clinical use. Circulation. 1996; 93:1043-65.

8. Liatis S, Alexiadou K, Tsiakou A, et al. Cardiac autonomic function correlates with arterial stiffness in the early stage of type 1 diabetes. Exp Diabetes Res. 2011;2011:957901.

9. Jaiswal M, Urbina EM, Wadwa RP, et al. Reduced heart rate variability is associated with increased arterial stiffness in youth with type 1 diabetes: the SEARCH CVD study. Diabetes Care. 2013;36:2351-8.

10. Nemes A, Takacs R, Gavaller H, et al. Correlations between aortic stiffness and parasympathetic autonomic function in healthy volunteers. Can J Physiol Pharmacol. 2010;88:1166-71.

11. European Society of Cardiology. Determinants of pulse wave velocity in healthy people and in the presence of cardiovascular risk factors: 'establishing normal and reference values'. Eur Heart J. 2010;31:2338-50.

12. American Diabetes Association. Cardiovascular disease and risk management. Diabetes Care. 2017;40:S75-87.

13. Tentolouris N, Liatis S, Moyssakis I, et al. Aortic distensibility is reduced in subjects with type 2 diabetes and cardiac autonomic neuropathy. Eur J Clin Invest. 2003;33:1075-83.

14. Meyer C, Milat F, McGrath BP, et al. Vascular dysfunction and autonomic neuropathy in Type 2 diabetes. Diabet Med. 2004;21:746-51.

15. American Diabetes Association. Classification and diagnosis of diabetes. Diabetes Care. 2017:40:S11-24.

16. World Medical Association declaration of Helsinki. Recommendations guiding physicians in biomedical research involving human subjects. JAMA. 1997;277:925-6.

17. Mancia G, De Backer G, Dominiczak A, et al. Guidelines for the management of arterial hypertension: the task force for the management of arterial hypertension of the European Society of Hypertension (ESH) and of the European Society of Cardiology (ESC). J Hypertens. 2007;25:1751-62.

18. Bos WJ, Verrij E, Vincent HH, et al. How to assess mean blood pressure properly at the brachial artery level. J Hypertens. 2007;25:751-5.

19. Newman AB, Siscovick DS, Manolio TA, et al. Ankle-arm index as a marker of atherosclerosis in the Cardiovascular Health Study. Cardiovascular Heart Study (CHS) Collaborative Research Group. Circulation. 1993;88:837-45

20. Young MJ, Boulton AJ, MacLeod AF, et al. A multicentre study of the prevalence of diabetic peripheral neuropathy in the United Kingdom hospital clinic population. Diabetologia. 1993;36:150-4.

21. Friedewald WT, Levy RI, Fredrickson DS. Estimation of the concentration of low-density lipoprotein cholesterol in plasma, without use of the preparative ultracentrifuge. Clin Chem. 1972;18:499-502.

22. Levey AS, Bosch JP, Lewis JB, et al. A more accurate method to estimate glomerular filtration rate from serum creatinine: a new prediction equation. Modification of diet in renal disease dtudy group. Ann Intern Med. 1992; 130:461-70.

23. Pauca AL, O'Rourke MF, Kon ND. Prospective evaluation of a method for estimating ascending aortic pressure from the radial artery pressure waveform. Hypertension. 2001;38:932-7.

24. Skrapari I, Tentolouris N, Perrea D, et al. Baroreflex sensitivity in obesity: relationship with cardiac autonomic nervous system activity. Obesity (Silver Spring). 2007;15:1685-93.

25. Gentile S, Bizzarro A, Marmo R, et al. Medial arterial calcification and diabetic neuropathy. Acta Diabetol Lat. 1990;27:243-53.

26. Cecelja M, Jiang B, Bevan $L$, Frost ML, et al. Arterial stiffening relates to arterial calcification but not to noncalcified atheroma in women. A twin study. J Am Coll Cardiol. 2011;57:1480-6.

27. MacFadyen RJ, Lim HS. Emergence of structural arterial stiffness in diabetes and the role of abnormalities of autonomic tone in the sequence of events. J Hum Hypertens. 2004;18:755-6.

28. Lacolley P, Glaser E, Challande P, et al. Structural changes and in situ aortic pressure-diameter relationship in long-term chemical-sympathectomized rats. Am J Physiol. 1995;269:H407-16.

29. Mattace-Raso FU, van den Meiracker AH, Bos WJ, et al. Arterial stiffness, cardiovagal baroreflex sensitivity and postural blood pressure changes in older adults: the Rotterdam Study. J Hypertens. 2007;25:1421-6.
30. Radaelli A, Castiglioni P, Balestri G, et al. Increased pulse wave velocity and not reduced ejection fraction is associated with impaired baroreflex control of heart rate in congestive heart failure. J Hypertens. 2010;28:1908-12.

31. Chesterton LJ, Sigrist MK, Bennett T, et al. Reduced baroreflex sensitivity is associated with increased vascular calcification and arterial stiffness. Nephrol Dial Transplant. 2005;20:1140-7.

32. Cecelja M, Chowienczyk P. Dissociation of aortic pulse wave velocity with risk factors for cardiovascular disease other than hypertension: a systematic review. Hypertension. 2009;54:1328-36.

33. Kollias A, Lagou S, Zeniodi ME, et al. Association of central versus brachial blood pressure with target-organ damage: Systematic review and metaanalysis. Hypertension. 2016;67:183-90.

\section{Submit your next manuscript to BioMed Central and we will help you at every step:}

- We accept pre-submission inquiries

- Our selector tool helps you to find the most relevant journal

- We provide round the clock customer support

- Convenient online submission

- Thorough peer review

- Inclusion in PubMed and all major indexing services

- Maximum visibility for your research

Submit your manuscript at www.biomedcentral.com/submit
) Biomed Central 